\title{
Contributing Ad Hoc Reviewers 2020
}

Rehabilitation Psychology publishes articles that capture the breadth of science and practice in rehabilitation psychology. To achieve this mission, we rely on a diverse group of individuals who serve as ad hoc reviewers. We are grateful to the following individuals for their generous commitment of time and effort for the 2020 volume of Rehabilitation Psychology. If you would like to join this group of individuals by serving as an ad hoc reviewer, please contact the editor at ehde@uw.edu.

$\begin{array}{ll}\text { Rachel Aaron } & \text { Ewa Domagała-Zyśk } \\ \text { Leah Adams } & \text { Mary Driscoll } \\ \text { Stephanie Danielle Agtarap } & \text { Jennifer Duchnick } \\ \text { May Albee* } & \\ \text { Herb Ames } & \text { Rosa Esteve Zarazaga } \\ \text { Diane Amstutz } & \text { Bella Etingen } \\ \text { Melissa Anderson } & \text { Delaney Evans* } \\ \text { Anne Arewasikporn } & \\ \text { James Arruda } & \text { Eerika Finell } \\ \text { Caitlyn Arutiunov* } & \text { Pamela Fitzpatrick } \\ \text { Kara Ayers } & \text { Amina Flowers* } \\ & \text { Martin Forchheimer } \\ \text { Anna Banik } & \text { Emily Foxen-Craft } \\ \text { Kathleen T. Bechtold } & \text { Katherine Parris Frey }\end{array}$

Jacob Bentley

Krista Best

Jagriti 'Jackie' Bhattarai

Malachy Bishop

John Parle Blake

Neil Bockian

Patrick Brice

Caitlin Campbell

Jane E. M. Carter

Fong Chan

Peii Chen

Chloe Chermette

Guillaume Chevance

I-Hua Chu

Nancy Ciccollela

Jillian Clark

Sarah W. Clark

Ashley N. Clausen

Olivio J. Clay

Ram Cnaan

Samuel Colbert*

Martha Combs

Kristin Jane Conover

Andrew Cook

Bridget Cotner

Laura Crocker

Willemien de Kleijn

Kyle Deane

Roxana Delgado

Nicole Ditchman

Mayra Juliana Galvis Aparicio
Rena Gatzounis
Michael Geisser
Helen Genova
Melita J. Giummarra
Jenna Goesling
Chantea D. Goetz
Jessica Graham
Joan Griffin
Elizabeth S. Gromisch

Elizabeth Hahn

Janette Hamilton

Kaitlin Amanda Harding

Richard Henry

Tracy Herring

Shivayogi V. Hiremath

Matthew Hocking

Amy Holley

Sigmund Hough

Summer Ibarra

Jeannette Iskander*

Amy Jak

Alicia M. January

Chad Johnson

Erica K. Johnson

Kai Karos

Minjung Kim
Lindsey Knowles

Megan Kramer

Denise Krch

Tracy Kretzmer

Dan Leibel $^{*}$

Irene W. Leigh

Kathleen Lemanek

Anthony H. Lequerica

Sheera F. Lerman

Allison Levine

Gloria Yuet Kwan Ma

Shelby Mann*

Shannon McCaslin

Lakeya McGill*

Grace McKee

Melody Mickens

Victor Molinari

Whitney Morean*

Helene Moriarty

Jeri Morris

Tony Morris

Carlyn Mueller*

Sara Mulroy

Chung Jung Mun

Andrew Nabasny*

Ruth Nelson

Sarah Nelson

Andrea Newman*

Thomas Novack

Anna S. Ord*

Deirdre O'Sullivan

Jaclyn Lennon Papadakis

Claudio Peter

Sonia Peterson

Kala Phillips

Lawrence Pick

Treven Curtis Pickett

Jennifer Pierce

Matthew Plow

Ruchika Shaurya Prakash

Mickeal Pugh
Amanda Raines

Michelle Roberts

David Rothman

Matthew E. B. Russell

Eric Scott

Danielle Shapiro

Christopher Shields

Marc A. Silva

Richard L. Skolasky

Beth Springate

Amy Starosta

Brocha Z. Stern

William Stiers

Kirk J. Stucky

John Andrew (Drew) Sturgeon

Lauren Stutts

Preeti Sunderaraman

Stacy Suskauer

Megan Sutter

Alicia Swan

Shane Norman Sweet

Elizabeth C. Thomas

Sarah Tlustos

Marta Tremolada

Callie Elizabeth Tyner

Ashley Vaillancourt

William Waked

Dustin Wallace

Erica Weber

Abigail Welch*

Daniel Whibley

Shelley Wiechman

Toni Williams

Meredith L. C. Williamson

Jennifer Wilson

Brent Womble*

Dalun Zhang

Maisa Ziadni

* Denotes Co-reviewer. 